\title{
HANDLING INSENSITIVITY IN MULTI-PHYSICS INVERSE PROBLEMS USING A COMPLEX EVOLUTIONARY STRATEGY
}

\author{
Jakub SAWicki, Maciej SMolka*, Marcin Łoś, Robert Schaefer \\ AGH University of Science and Technology, al. Mickiewicza 30, 30-059 Krakow, Poland \\ *Corresponding author: smolka@agh.edu.pl
}

\begin{abstract}
In this paper we present a complex strategy for the solution of ill posed, in-verse problems formulated as multiobjective global optimization ones. The strategy is capable of identifying the shape of objective insensitivity regions around connected components of Pareto set. The goal is reached in two phases. In the first, global one, the connected components of the Pareto set are localized and separated in course of the multi-deme, hierarchic memetic strategy HMS. In the second, local phase, the random sample uniformly spread over each Pareto component and its close neighborhood is obtained in the specially profiled evolutionary process using multiwinner selection. Finally, each local sample forms a base for the local approximation of a dominance function. Insensitivity region surrounding each connected component of the Pareto set is estimated by a sufficiently low level set of this approximation. Capabilities of the whole procedure was verified using specially-designed two-criterion benchmarks.
\end{abstract}

Key words: Multi-objective optimization, Evolutionary algorithm, Iinverse problem

\section{INTRODUCTION}

\subsection{Ill-posedness of Inverse Problems}

The ill-posedness is a common feature of optimal design problems appearing in technology (e.g., lens design (Isshiki et al., 2006), environmental engineering (e.g., Rainfall-Runoff Models calibration (Duan et al., 1992), etc. In this paper we consider coefficient Inverse Problems (IPs) (see e.g. Tarantola, 2005) formulated as the global optimization problems (GOPs), such as those arising in the cancer diagnosis (see e.g. Paruch \& Majchrzak (2007)) or the oil and gas resource investigation (see e.g. Smołka et al., 2015a). Such problems typically suffer from several types of ill-posedness. One of the most serious is the insensitivity with respect to particular parameter changes. The insensitivity is manifested in subregions or manifolds surrounding global or local minimizers where the objective is constant or exhibits very small variation. To raise the difficulty, the insensitivity may be accompanied by multimodality, i.e., the objective may possess many glob- al or local minimizers with disconnected insensitivity sets.

\subsection{Solving Inverse Problems expressed as a multiobjective optimization}

In case of inverse problems formulated as the global optimization ones, the objective is defined as the misfit between an actual measurement $d$ of the studied phenomenon and the result of the simulation $u(\omega)$. Simulations are performed using a mathematical/computer model and its parameters $\omega$ which form decision variables. If the phenomenon under consideration is complex, i.e. it is composed of several coupled physical and information processes, it is possible to increase the amount of information by observing multiple parallel measurements of different data which give rise to many misfits and puts the problem into the area of multi-objective Pareto analysis. Such approach was already studied in our earlier papers, e.g. (Gajda-Zagórska et al., 2017; GajdaZagórska et al., 2015; Gajda-Zagórska, 2015). 
To this end, let us consider the set of $n$ physical processes with states $u_{i}(\omega) \in V_{i}, i=1, \ldots, n$, associated with the analyzed phenomenon. We assume that all processes are profiled with the same unknown parameter $\omega \in \mathcal{D}$. We restrict ourselves to the cases in which $\omega$ is a discrete representation of a physical quantity not necessarily discrete in its nature (e.g. heat conductivity). As a result, the admissible set of parameters $\mathcal{D}$ is embedded in a finite dimensional metric space, in most cases $\mathcal{D} \subset \mathbb{R}^{k}$ for an integer $k \geq 1$. The state spaces $V_{i}$ are typically appropriate Sobolev spaces and $A(u(\omega))$ is the relevant system $A_{i}\left(u_{i}(\omega)\right)=0, i=1, \ldots, n$ of differential equations representing forward problems. The state coordinates $u_{i}(\omega), i=1, \ldots, n$ are partially measurable and we denote by $d ; d_{i} \in \mathcal{O}^{i}$ the measurement vector, where $O^{i}$ are the observation spaces specific to each physics. We consider the misfit operator:

$$
\begin{aligned}
& f(d, u(\omega))=f^{1}\left(d^{1}, u^{1}(\omega)\right), \ldots \ldots, f^{n}\left(d^{n}, u^{n}(\omega)\right) \\
& \mathcal{O} \times \mathcal{D} \ni\left(d^{i} u^{i}(\omega)\right) \rightarrow f^{i}\left(d^{i} u^{i}(\omega)\right) \in \mathbb{R}_{+}, i=1, \ldots, n
\end{aligned}
$$

where each coordinate $f^{i}$ represents the particular physics. The multi-objective optimization problem associated with multi-physics IP consists of finding the Pareto set $\mathcal{P S} \subset \mathcal{D}$, such that all $\omega \in \mathcal{P S}$ satisfy:

$$
\min _{\omega \in \mathcal{D}}\{f(d, u(\omega)) ; A(u(\omega))=0\}
$$

where the minimization is understood with respect to Pareto dominance relation, see e.g. (Miettinen, 1999).

\subsection{Insensitivity regions in multiobjective optimization}

To investigate insensitivity regions we introduce an auxiliary scalar function $\Psi: \mathcal{D} \rightarrow \mathbb{R}$ which measures the degree of domination among points in $\mathcal{D}$. Let $\mathcal{P F} \subset \mathbb{R}^{n}$ denote the Pareto front of our optimization problem. Function $\psi$ is defined as:

$$
\Psi(\omega)=d(\mathcal{P} \mathcal{F}, F(\omega)) \equiv \inf _{y \in \mathcal{P} \mathcal{F}}\|F(\omega)-y\|
$$

where $F: \mathcal{D} \rightarrow \mathbb{R}^{n}$ is a mapping of the decision space to the criteria space, i.e.:

$$
F(\omega)=f(d, u(\omega)), \quad \text { where } A(u(\omega))=0
$$

Function $\Psi$ is continuous, provided criteria functions $f_{i}$ are, and its zero set is precisely the Pareto front $\mathcal{P} \mathcal{F}$. Its level sets:

$$
L_{\epsilon}(\Psi)=\{\omega \in \mathcal{D}: \Psi(\omega)<\epsilon\}
$$

are therefore open neighborhoods of $\mathcal{P} \mathcal{S}$ in $\mathcal{D}$. For a suitably low threshold value $\epsilon$, connected components of such a set can be then interpreted as the low-sensitivity regions of the problem

\subsection{State of the art}

The most classical way of improving illposedness of a GOP is the Tikhonov regularization (Tikhonov et al., 1995), which consists in completing the misfit by the convex suplement. However, this method is restricted to the single-criterion problems which are conditionally well posed in sense of Tikhonov, see again (Tikhonov et al., 1995).

If the misfit contains a noise which makes its behaviour difficult to analyze, the Uncertain Optimization (UO) and Robust Optimization (RO) can be applied. In these methods the objective $f(\omega, \xi)$ depends on the deterministic decision $\omega$ and a random variable $\xi$, which represent noise, see e.g. (Bertsimas \& Brown, 2009; Jin \& Branke, 2005). Usually, each component of $\xi$ is associated with a design constant, a design value which isn't modified by the optimization algorithm, but which real-world realization is subject to uncertainty. As a consequence, the misfit is a random function too, and its realization might take different values for consecutive calls of the computing routine at the same decision $\omega$. It is typically assumed by $\mathrm{UO}$ and RO analysis, that there exists an accurate objective $\bar{f}(\omega)$ which corresponds with its noisy version, and it can be approximated by its statistics, e.g. $\bar{f}(\omega) \approx E[f(\omega, \xi)]$. Most often it is also assumed that the optimization problem imposed by $\bar{f}(\omega)$ is well posed. If some information about the noise $\xi$ are available, it is possible to compute some statistic of minimizers or try to evaluate the subsets of the admissible domain in which minimizer might occur with a suffciently large probability.

The problems of robust optimization are usually solved for single-objective cases. However, there are also attempts at taking RO into account in the multiobjective problems. One such example is described by Gunawan and Azarm (2005), where a concept of worst-case sensitivity region (in the space of design constants) is proposed. It is a region, on which the objective values change within accepted bounds so, the larger the region, the more robust the design is. A constraint on the radius of such region is imposed, so that solutions which do not meet it are removed during the optimization process, yielding a Pareto front of robust solutions. 
The uncertainty handling in multi-objective optimization problem by using Evolutionary Algorithm with the NSGA selection was mentioned by Petrone et al. (2013).

If the local misfit insensitivity is a result of the serious lack of information e.g. caused by the restricted observability or/and measurability, than illposedness is irrecoverable, and could not be removed by any stochastic or deterministic misfit regularization.

In such cases the information about all connected components of the set $L_{\epsilon}(\Psi)$ should be delivered to the user.

\subsection{The guideline of the paper}

The main contribution of this paper is a multiphase stochastic method of building a discrete approximation of $L_{\epsilon}(\Psi)$. The first phase of the method is a Hierarchic Memetic Strategy (HMS) equipped with the NSGA-II selection operator and a repository. Its aim is to detect the connected components of the (approximate) Pareto set. NSGA-II is based on a rank function which can be treated as an analogue to $\psi$. Then, points with suffciently low rank form the input to the second phase. In this phase a single-population evolutionary algorithm equipped with a special multiwinner-voting-type selection operator is executed separately for the individuals belonging to each connected component of the Pareto set approximation $L_{\epsilon}(\Psi)$. The aim of this phase is to approximate the shape of low-rank sets, and in consequence, level sets $L_{\epsilon}(\Psi)$. Finally, the results of the second phase form the basis for an approximation of $\psi$, which results in boosting the accuracy of the approximation of $L_{\epsilon}(\Psi)$.

\section{THE STRATEGY}

The overall control flow of the strategy is presented in figure 1 . The actions shown in the diagram represent the strategy phases, which shall be described in subsections of this section.

\subsection{Hierarchic Memetic Search as a global phase}

The global phase of our strategy is based on the Hierarchic Memetic Strategy (HMS) framework allowing the effective stochastic search for multimodal GOPs. The core of this framework was mentioned in (Gajda-Zagórska et al., 2017) and was broadly described in the monograph (Smołka, 2015), so we describe here only crucial HMS steps and ideas.



Fig. 1. Overall control flow of the described strategy (UML activity diagram).

The HMS creates and processes a tree of demes. HMS tree has a restricted depth $m<+\infty$ and usually the maximum number of child demes at each particular level is also restricted. Each deme of the maximum order $m$ will be called the leaf-deme.

Demes evolve according to specific selection, mixing and succession rules. Typically, HMS demes of the same order use the same encoding and the same evolutionary engine. The total HMS structure evolves in steps called metaepochs, while demes may perform more than one step of evolution during single metaepoch.

At the end of a metaepoch, each deme except the leaf-demes, can sprout a new child deme by sampling its new population around the best fitted individual. Sprout is blocked if the new deme lies too close to an already sprouted one at the same level of HMS tree.

The HMS deme structure and dynamics allow for fast encountering the promising regions in an admissible domain and locating non-overlapping leafdemes there.

The second leading HMS idea is profiling the search accuracy, typically lowest at the root and low order demes and maximum at leaf-demes. Frequently, the cost of the misfit evaluation depends monotonously on the required accuracy in the engineering inverse problems. In such case the HMS framework works especially economically, finding cheaply the promising regions (e.g. basins of attractions of minimizers or regions of insensitivity) and performing the accurate, expensive computations only there.

\subsection{Non-dominated Sorting selection}

The most popular evolutionary approach for multi-objective optimization called Multi-Objective Evolutionary Algorithm (MOEA) beside the common mixing operations (crossover and mutation) uses the special kind of selection called Nondominated Sorting operator (NS selection), see e.g. (Fonseca \& Fleming, 1993). NS selection is based 
on the set of ranks being its "domination" level with respect to all individuals in the current population e.g. $\operatorname{rank}(\omega)=0$ if $\omega$ is not dominated by any other individual from the current population, generally, $\operatorname{rank}(\omega)=k \geq 0$ if $\omega$ is dominated exactly by $k$ other individuals from the current population. Typically, MOEA also maintains a repository $\mathcal{R}$ in which the non-dominated individuals already found are gathered.

We use NS selection at each HMS level in the global phase of the presented strategy which leads to identify the insensitivity regions surrounding the Pareto set associated with an IP. For now, the repository $\mathcal{R}$ was input only the individuals from the leafdemes (the highest order demes). Individuals stored in the repository memorize their deme of origin identifiers. In contradiction to the MOEA, the repository will gather individuals with ranks smaller or equal to the particular, small integer $k>0$. The reason of attaching "weakly" dominated points to the repository is to collect broader information of the MOEA fitness behavior near the Pareto set.

The repository was updated after each metaepoch. First, individuals from all leaf-demes active in the last metaepoch were added to the repository. Next, all individuals $\omega \in \mathcal{R}$ which have $\operatorname{rank}(\omega)>k$ with respect to other repository members were removed.

\subsection{Global phase dynamics}

The HMS starts with a single population called root-deme. After each metaepoch, all demes, except the leaves, try to sprout a single child-deme. An empty repository is introduced when the first leafdeme is sprouted.

We use a simple termination condition of the efficiency type with two variants. The weaker of the two stops the strategy if the non-dominated part of the repository stays constant for a fixed number of epochs. The stronger termination condition becomes active if the whole repository does not change for a fixed number of epochs.

After HMS is terminated, the repository is divided into clusters of individuals originating from different demes. The leaf-demes are almost always highly concentrated, i.e., it is very unlikely that such a deme covers more then one connected component of the Pareto set. In fact, such a situation hardly ever appears in real computations. As a consequence, a density clustering algorithm run on the final repository would not provide any more information.
On the other hand, multiple leaf-demes may be located in the single connected component of the Pareto set. Such clusters are merged, using the hillvalley algorithm (Ursem, 1999). Finally, the global phase returns a family of clusters gathering individuals located in different, separated, connected parts of the Pareto set and their topological neighborhoods. Such family is then passed to the next phase of the strategy.

\subsection{Local phase activities}

The local phase activities lead to obtain the approximation of the shape of the insensitivity regions surrounding the connected parts of Pareto sets. The activities are performed in parallel for each cluster received from the global phase.

For each cluster, we run two epochs of the evolutionary algorithm with multiwinner (MW) selection (Łoś et al., 2017; Sawicki et al., 2018). The MW evolution uses standard mixing operators: normal mutation and arithmetic crossover. The fitness is based on the ranks obtained by non-dominated sorting, as in the global phase.

MW selection uses the utility function which describes the "utility" of each individual in the population gives to other individuals. It is meant to promote good fitness values while also valuing nearby individuals. Based on it, the multiwinner election is run. The function is defined as follows in (Loś et al., 2017):

$u_{i}^{p}\left(x^{(j)}\right)=\frac{h\left(f\left(x^{(j)}\right)\right)}{1+d\left(x^{(i)}, x^{(j)}\right)}$

where: $h(x)=1 /(1+x)$ - a reversal function, $d$ - the Euclidean distance, $x^{(i)} \in \mathcal{D}$ - a member of a population.

In the first epoch of MW evolution we use $h$ function defined by equation (6). The resulted population becomes distributed more uniformly inside the analyzed connected part of the insensitivity region.

In the second MW epoch we use a modified, "inverse" utility function. We substitute $h$ with $h^{\prime}(x)$ $=x$ forming a family of utility functions $\left(u_{i}^{p}\right)^{\prime}$. Because the fitness is subject to minimization, such modified utility function will favor individuals with worse fitness, spreading the individuals of the population. The random sample (population) obtained in the second MW epoch will spread to the neighborhood of the first epoch population. It is necessary to properly approximate the fitness function at the bor- 
der of the insensitivity region. Otherwise, the approximation would appear flat, and so the level set of such approximation would be of no use.

Approximation of the insensitivity region is constructed as a level set of a local approximation of the rank function, built using set $P$ of all the points produced in the first and second MW epoch and those inherited from the cluster. In case the insensitivity regions are large compared to the domain and not very numerous, this approach may be simplified by replacing the local approximation by a global one on the whole domain without a significant loss of accuracy. This approximation is created in two steps: first, a continuous linear Lagrange interpolation $\tilde{r}$ is constructed on a region $\Omega$ from the Delaunay triangulation of $P$. Then $\tilde{r}$ is continuously extended to $Q$ - the minimal hyperrectangle containing $\Omega$. Let $\mathcal{V}$ denote the space of $\mathrm{B}$-splines defined on $Q$, i.e. the tensor product of spaces of 1D B-splines with fixed degree $p \geq 1$ and knot vectors. Then $\mathcal{V} \subset L^{2}(Q)$ and elements of $\mathcal{V}$ are globally at least $C^{1}$. Finally, a smooth approximation $\bar{r}$ of the rank function is defined as the $L^{2}$-projection of $\tilde{r}$, i.e. the unique element of $\mathcal{V}$ with minimal distance to $\tilde{r}$ in $L^{2}$ norm:

$$
\mid \bar{r}-\tilde{r}\left\|_{L^{2}}=\inf _{v \in \mathcal{V}}\right\| v-\tilde{r} \|_{L^{2}}
$$

Such an element of $\mathcal{V}$ can be effciently computed as a solution of certain linear system using the Alternating Direction Solver (ADS) (cf. Gao \& Calo, 2014) thanks to the tensor product structure of the chosen basis. For more details on the employed approximation method, we refer to (Sawicki et al., 2018).

In addition, we compare this approach with a standard method - Simple Kriging with constant trend and exponential semivariogram model (Jin, 2005).

\section{EXPERIMENTS}

We applied the described complex strategy to two two-criterion multimodal benchmark GOPs. The objectives in these problems were constructed in such a way that their Pareto sets have the following features:

they can be determined analytically;

- they are "thick", i.e., they have positive Lebesgue measure.

The first feature provides the reference level for checking the abilities of our strategy. The second one mimics analogous features of ill-posed inverse problems formulated as GOPs.

To determine the quality of insensitivity region approximation we compare it with the known exact region by computing the Hausdor distance between these sets. Let $A ; B \in X$ be subsets of a metric space $(X ; d)$, let:

$$
\delta(A, B)=\operatorname{supinf}_{x \in A} d(x, y)
$$

The Hausdor distance $d_{H}(A ; B)$ is then defined as:

$d_{H}(A, B)=\max \{\delta(A, B), \delta(B, A)\}$

\subsection{First benchmark problem}

Let $\mathcal{D}=[0 ; 10]^{2}$ and let us define:

$g_{\left(x_{1}, x_{2}\right)}^{\left(r_{1}, r_{2}\right)}\left(x_{1}, x_{2}\right)=1-2^{-\left(\frac{x_{1}-x_{1}^{0}}{r_{1}}\right)^{2}-\left(\frac{x_{2}-x_{2}^{0}}{r_{2}}\right)^{2}}$

Then $\mathrm{g}$ is a smooth function with values between 0 and 1 with $g\left(x_{1}^{0}, x_{2}^{0}\right)=0$. Objective function $F^{1}=$ $\left(f_{1}^{1}, f_{2}^{1}\right): \Psi: \mathcal{D} \rightarrow \mathbb{R}^{2}$ is given by:

$$
\begin{aligned}
& f_{1}^{1}(\mathbf{x})=\sigma\left(g_{(2,2)}^{(0.5,1.0)}(\mathbf{x}) \cdot g_{(8,5)}^{(0.5,1.0)}(\mathbf{x})\right) \\
& f_{2}^{1}(\mathbf{x})=\sigma\left(g_{(3,3)}^{(1.0,0.5)}(\mathbf{x}) \cdot g_{(7,5)}^{(1.0,0.5)}(\mathbf{x})\right)
\end{aligned}
$$

where:

$$
\sigma(t)=\max \{2 t-1,0\}= \begin{cases}2 t-1 & \text { if } t<\frac{1}{2} \\ 0 & \text { otherwise }\end{cases}
$$

Components $f_{1}^{1}, f_{2}^{1}$ of the objective are presented in figure 2. The functions are chosen in such a way that the Pareto set consists of two connected components situated in the vicinity of intersections of component functions' global minima (see figure $3)$.

\subsection{Second benchmark problem}

A weak side of the first benchmark is that its Pareto front (not Pareto set) is trivial, i.e., it reduces to singleton $\{(0,0)\}$. The second benchmark lacks this drawback: in this case neither Pareto front nor Pareto set is trivial. This time the domain is $\mathcal{D}=$ $[0,1]^{2}$. Objective function $F^{2}=f_{1}^{1}, f_{2}^{1}: \mathcal{D} \rightarrow \mathbb{R}^{2}$ is given by:

$$
\begin{aligned}
& f_{1}^{2}(\mathbf{x})=x_{1}+\max \left\{\left|x_{2}-0.5\right|-0.1,0\right\} \\
& f_{2}^{2}(\mathbf{x})=1-x_{1}+\max \left\{\left|x_{2}-0.5\right|-0.1,0\right\}
\end{aligned}
$$




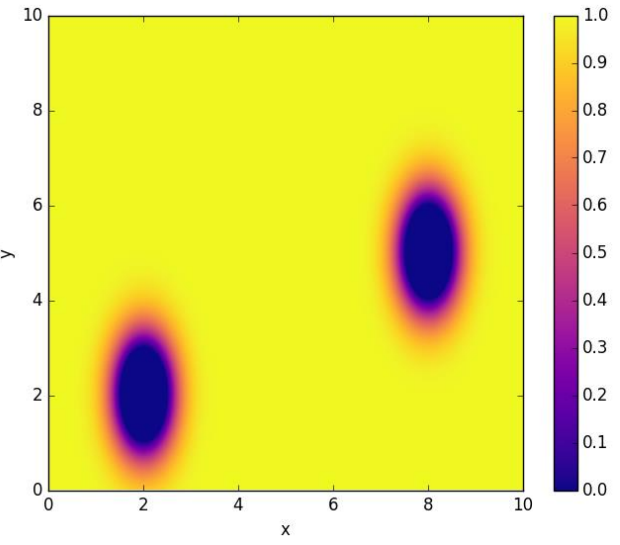

a)

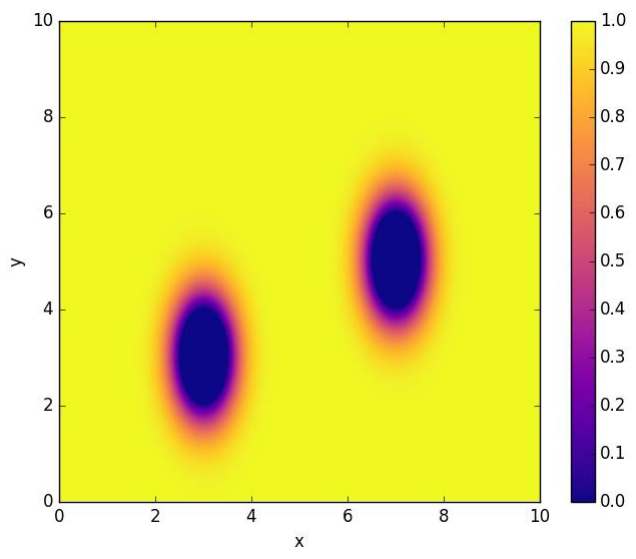

b)

Fig. 2. Objective functions $f_{1}^{1} \quad(a)$ and $f_{2}^{1}(b)$ in the first benchmark.

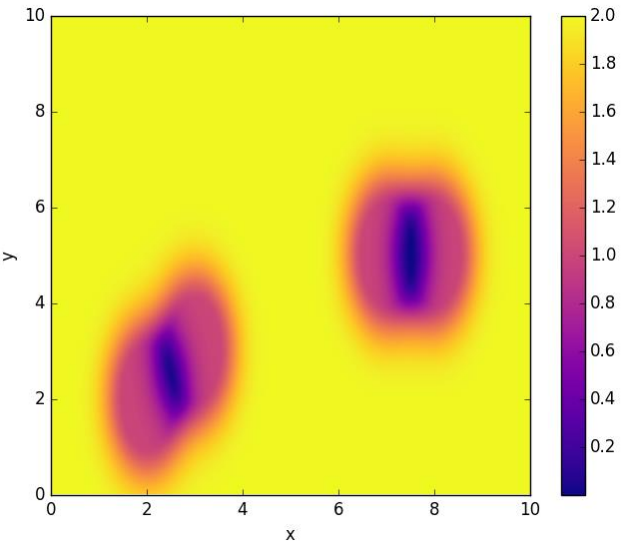

Fig. 3. Overall control flow of the described strategy (UML activity diagram).

The Pareto front in this case is the segment of the line $x_{1}+x_{2}=1$ contained in $\mathcal{D}$ (see figure $4 \mathrm{a}$ ). The Pareto set is the rectangle $[0,1] \times[0.4,0.6]$ (see figure $4 b)$.

\subsection{Strategy configuration}

In the experiments we used two three-level HMS configurations differing in the evolutionary engine used in demes:
- HMS-SSS: demes at all levels powered by the Simple Evolutionary Algorithm (SEA) with the Nondominated Sorting selection;

- HMS-SSN: follows the same configuration as in the previous HMS-SSS case, except for that demes at the leaf level evolved along the lines of NSGA-II strategy, see e.g. (Deb et al., 2002).

a)
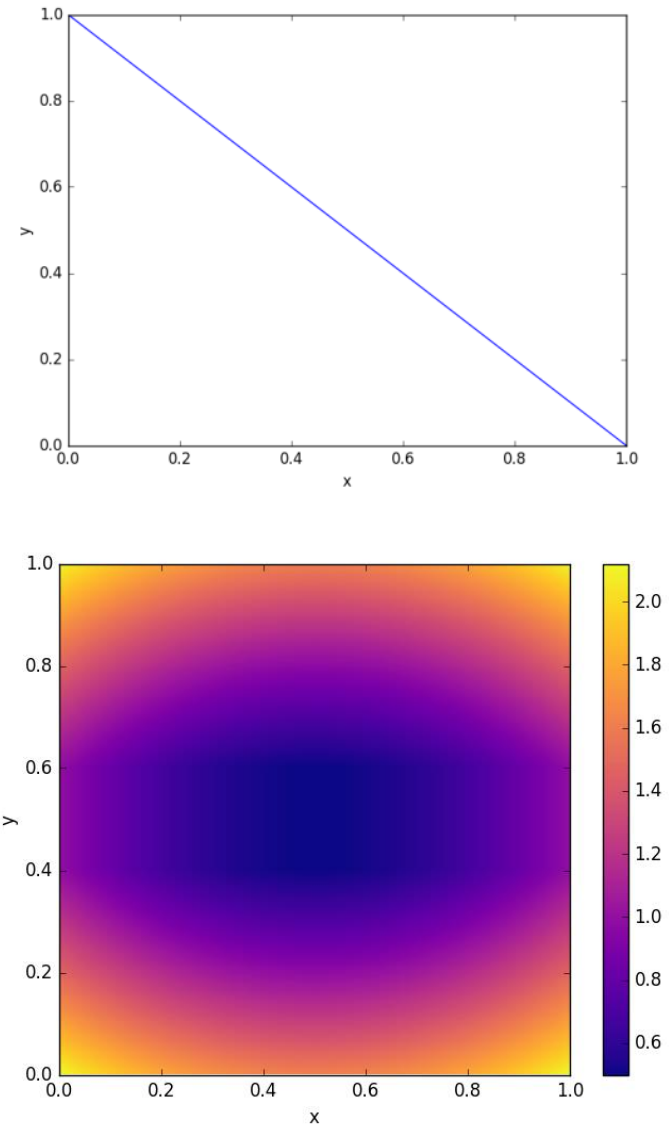

Fig. 4. Second benchmark: Pareto front (a) and squared norm $\left\|F^{2}\right\|^{2}$ of the objective $(b)$.

The first configuration was commonly used in our previous experiments (cf. Smołka et al., 2015a; Smołka et al., 2015b; Gajda-Zagórska et al., 2017), the second one engages the widely used multiobjective evolutionary algorithm - NSGA-II. Both configurations were executed 10 times on each benchmark. The global phases were stopped after performing 10000 evaluations of the objective functions. Then both above-described MW local phases ("direct" and "inverse") were executed in each case. The archive retained candidate solutions from the global phase with rank less than or equal to $k=2$. The whole final populations of both MW phases were also put into the archive. Afterwards, $L^{2}$ and Kriging approximations (see above) of rank functions were constructed for those final populations. As the final population highest rank varied significantly not only between benchmarks but also be- 
tween particular executions, we modified the obtained approximate rank function using the nonlinear scaling function.

$$
s(x)=1-\frac{1}{1+x}
$$

This made it fair to use the same cutting level for all runs of both benchmarks. The final Pareto set shape approximations were thus obtained as the level sets for the scaled approximate rank functions at level $\epsilon=0.2$.

\subsection{Results for the first benchmark}

As the computational domain is relatively small and the well-known Pareto set components are close to each other, we decided to use the global approximation approach in the last phase of the strategy. The obtained Hausdor distance statistics are presented in table 1 for HMS-SSS and in table 2 for HMSSSN. Both strategies managed to detect both components of the Pareto set in every run. $L^{2}$ approximation turned out to be significantly more accurate than Kriging in this case, especially when combined with HMS-SSS. Additionally, we show in figures 5a and 7 the results of a successful execution of HMS-SSS, and in figures $5 \mathrm{~b}$ and 8 the results of a relatively weak execution of this strategy. Figures $6 \mathrm{a}$ and $6 \mathrm{~b}$ illustrate analogous results for HMS-SSN. The quality of final results depend highly on the second ('inverse') MW phase of the whole strategy. The global phase produces hardly any dominated points. The first MW phase fills in detected areas appropriately, but it is only the second MW phase that can surround these areas with a significant nondominated 'blanket' that would allow the approximation phase to catch the shape of true Pareto set component. As it can be seen, the Kriging approximation is particularly sensitive to the extent of this blanket: in figure $5 \mathrm{~b}$ the upper-right component was extended extraordinarily, in figure $6 \mathrm{~b}$ we have even an additional false component of Pareto set. These artifacts are apparent result of significant gaps in the non-dominated blanket. The gaps can be seen in figure 8 where the points obtained from the MW phases are shown as red dots. Note that the effects of the gaps are significantly milder if $L^{2}$ approximation is used.

Table 1. HMS-SSS: Hausdorff distances in the first benchmark.

\begin{tabular}{|c|c|c|}
\hline Approximation & $L^{2}$ & Kriging \\
\hline Average distance & 0.36476 & 0.60703 \\
Distance std. dev. & 0.04052 & 0.870822 \\
\hline
\end{tabular}

Table 2. HMS-SSN: Hausdorff distances in the first benchmark.

\begin{tabular}{|c|c|c|}
\hline Approximation & $L^{2}$ & Kriging \\
\hline Average distance & 0.49464 & 0.69073 \\
Distance std. dev. & 0.13170 & 0.87405 \\
\hline
\end{tabular}

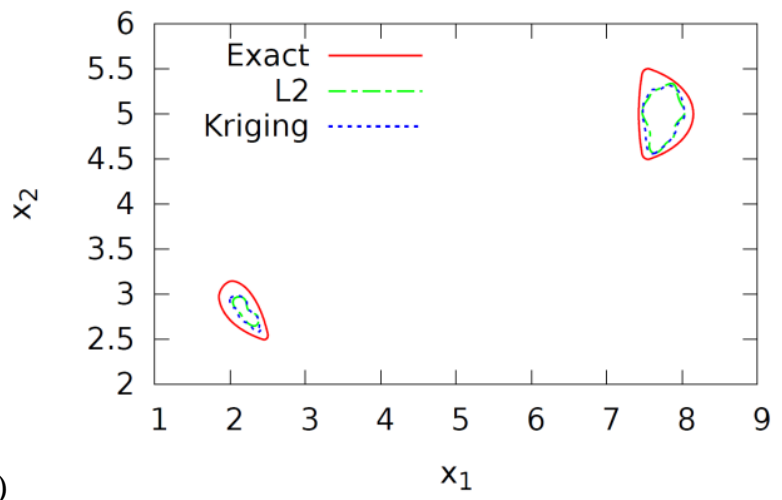

a)



Fig. 5. HMS-SSS in first benchmark: Pareto set approximations for a'good' run (a) and a'bad' run (b).
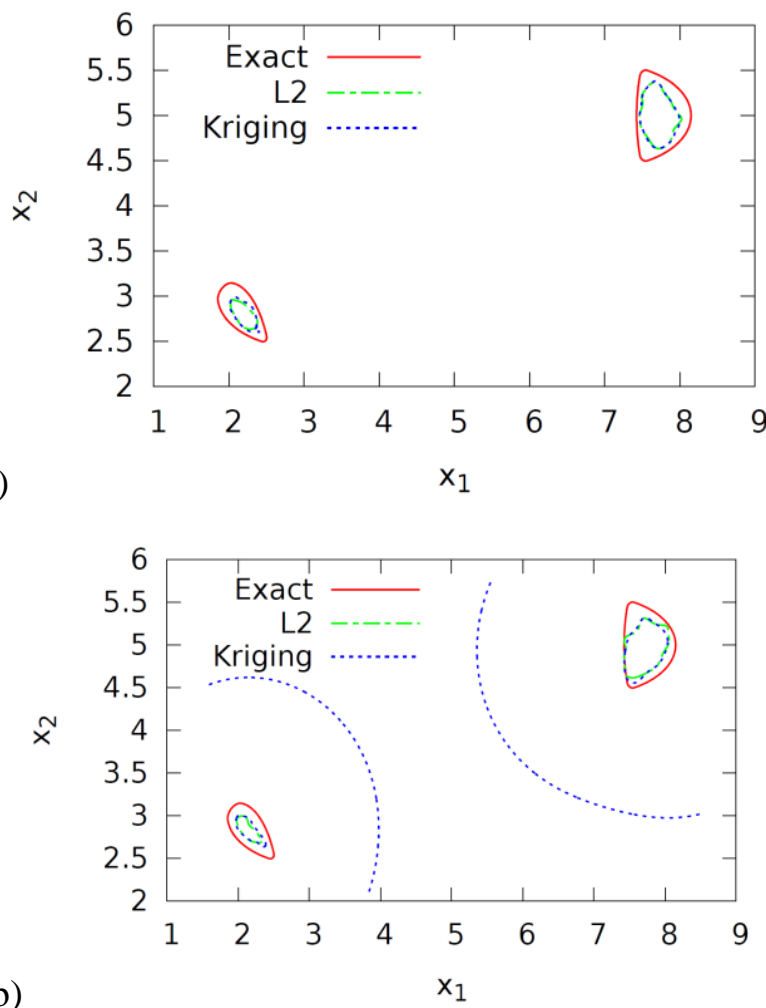

Fig. 6. HMS-SSN in first benchmark: Pareto set approximations for a 'good' run (a) and a'bad' run (b). 


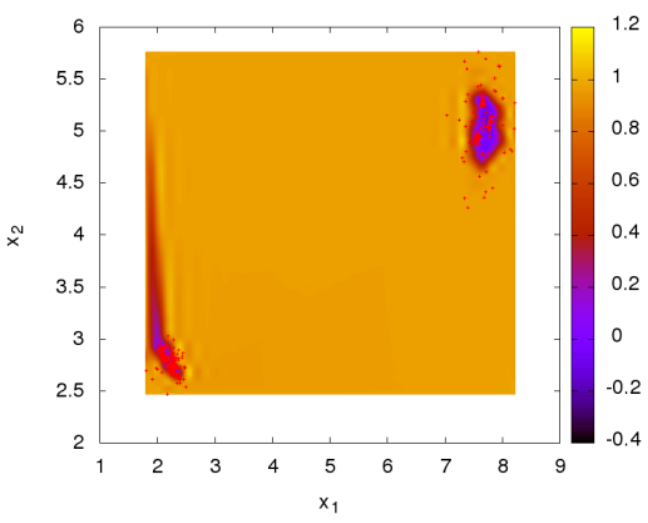

a)

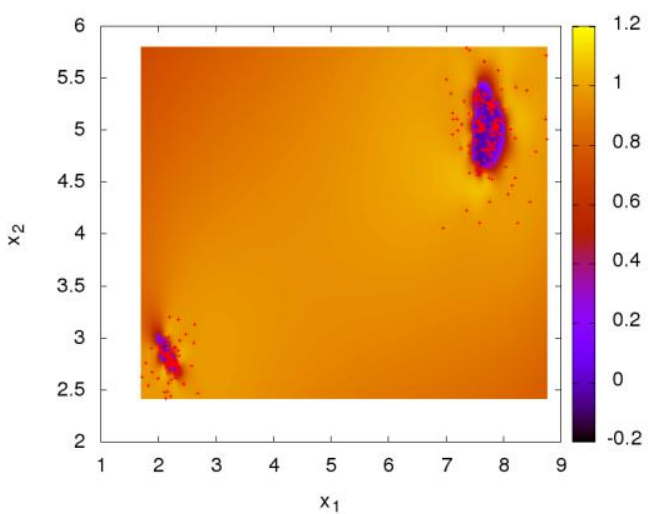

b)

Fig. 7. Rank approximations in HMS-SSS 'good' run for $L^{2}$ approximation (a) and Kriging approximation (b).

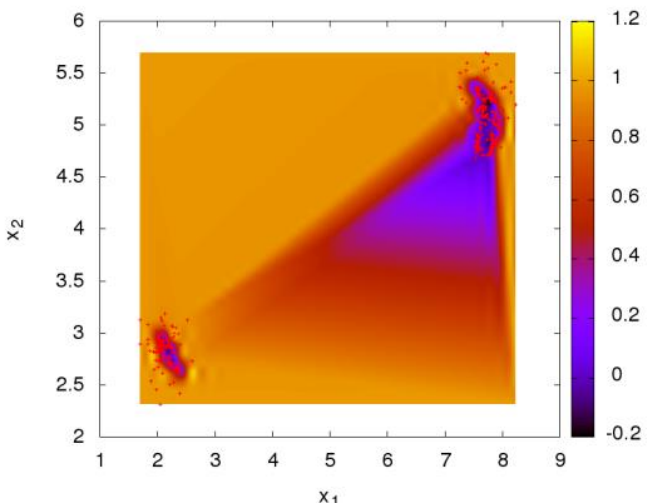

a)

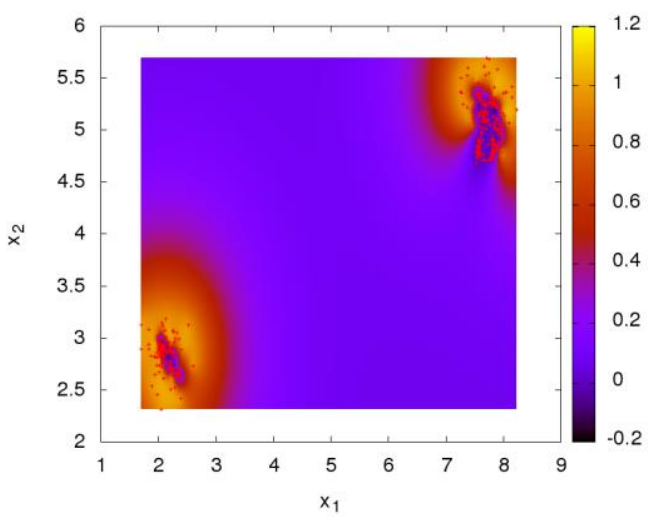

b)

Fig. 8. Rank approximations in HMS-SSS 'bad' run for $L^{2}$ approximation (a) and Kriging approximation (b).

\subsection{Results for the second benchmark}

As before, the obtained Hausdor distance statistics are presented in table 3 for HMS-SSS and in table 4 for HMS-SSN. Also as in the previous subsection, we show in figures $9 \mathrm{a}$ and $9 \mathrm{~b}$ the results of a successful and a weak execution of HMS-SSS, and in figures $10 \mathrm{a}$ and $10 \mathrm{~b}$ the results of a relatively good and a relatively bad run of HMS-SSN. In this case we do not observe significant differences either between evolutionary engines or between approximation methods. A big part of artifacts observed in the figures is related to the shortage of points provided by global and MW phases near the domain boundary. Besides, the non-dominated blanket has a proper extent in virtually all runs resulting in a fair quality of Pareto set approximation independently upon the approximation method.

Table 3. HMS-SSS: Hausdorff distances in the second benchmark.

\begin{tabular}{|c|c|c|}
\hline Approximation & $L^{2}$ & Kriging \\
\hline Average distance & 0.39259 & 0.39079 \\
Distance std. dev. & 0.0063836 & 0.0074297 \\
\hline
\end{tabular}

Table 4. HMS-SSN: Hausdorff distances in the second benchmark.

\begin{tabular}{|c|c|c|}
\hline Approximation & $L^{2}$ & Kriging \\
\hline Average distance & 0.38975 & 0.38738 \\
Distance std. dev. & 0.0050023 & 0.0063584 \\
\hline
\end{tabular}

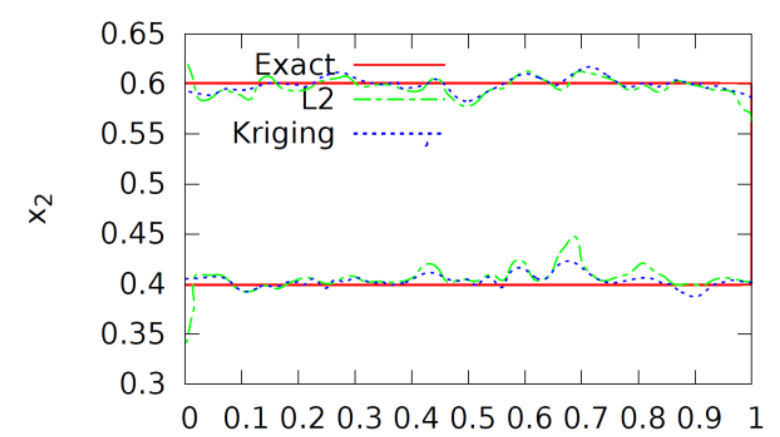

a)

$\mathrm{x}_{1}$

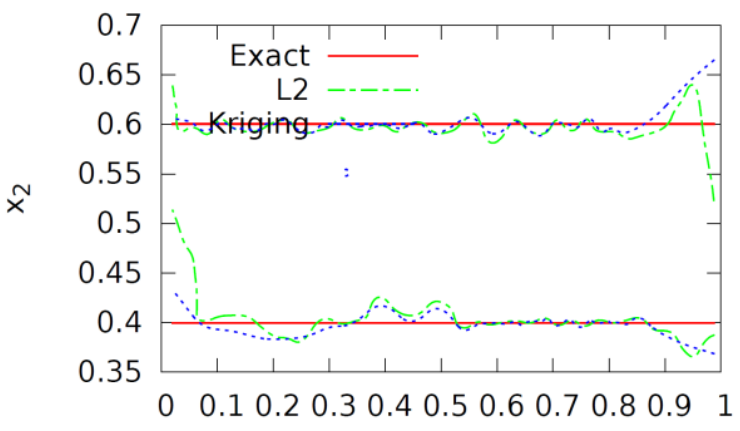

b)

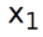

Fig. 9. HMS-SSS in second benchmark: Pareto set approximations for a 'good' run (a) and a 'bad' run (b). 


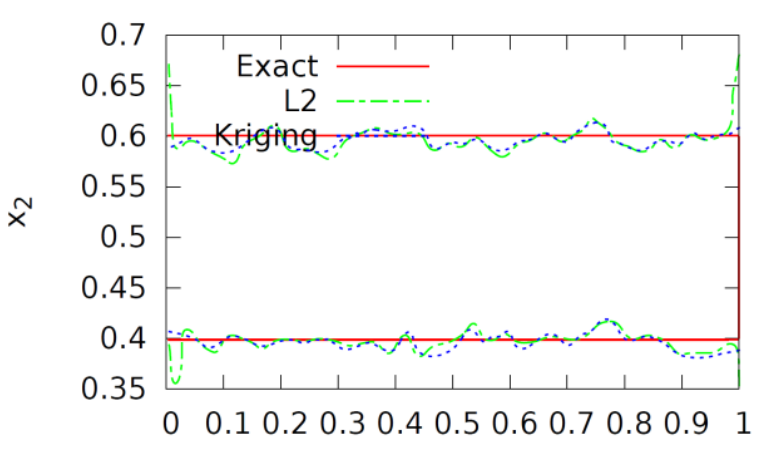

a)

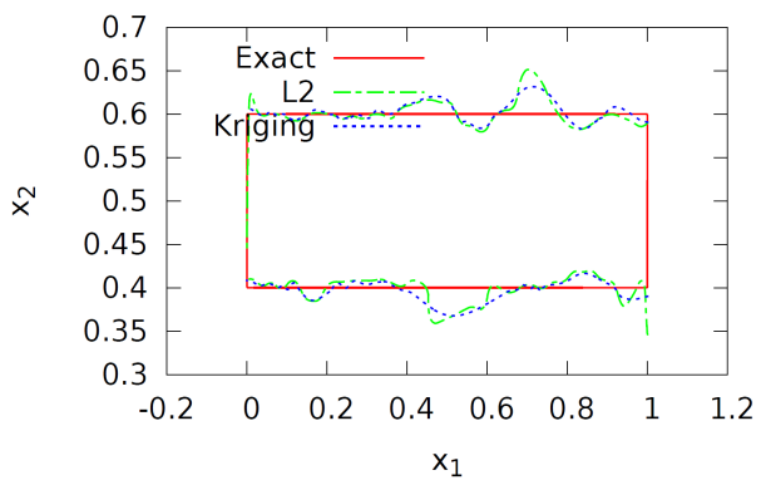

Fig. 10. HMS-SSN in second benchmark: Pareto set approximations for a 'good' run (a) and a 'bad' run (b).

\section{CONCLUSIONS}

The presented complex strategy proved its capabilities in identifying the shape of insensitivity regions around solutions of multimodal multiobjective problems. The global phase of the algorithm successfully detects basins of attraction of all the global minima. Afterwards, in two special evolutionary phases, the detected areas are properly filled and surrounded with an appropriate 'blanket' of nondominated points. Both activities are key steps of the whole strategy, because their successful execution forms a solid base for the following approximation phase. The obtained results show that the presented strategy equipped with $L^{2}$ approximation produces good-quality approximation of shapes of insensitivity sets in all cases. The Kriging method in turn can produce artifact sets when the second MW phase does not provide a gapless non-dominated blanket. We expect that this issue can be negotiated by, e.g. using two or more subsequent inverse MW phases.

In this paper, for illustrative reasons we evaluated the strategy by means of twoobjective twodimensional benchmarks only. In the future we plan to test it using more-dimensional benchmarks on one hand and some real-world engineering multi objective inverse problems, like the several-frequency magnetotelluric problem, on the other hand.

\section{ACKNOWLEDGEMENTS}

The work presented in this paper has been partially supported by Polish National Science Center grants no. DEC-2015/17/B/ST6/01867 and by the AGH statutory research grant no. 11.11.230.124.

\section{REFERENCES}

Bertsimas, D., Brown, D.B., 2009, Constructing uncertainity sets for robust linear optimization, Operational research, 57, 1483-1495.

Deb, K., Pratap, A., Agarwal, S., Meyarivan, T., 2002, A fast and elitist multiobjective genetic algorithm: NSGA-II, IEEE Transactions on Evolutionary Computation, 6 (2), 82-197.

Duan, Q., Sorooshian, S., Gupta, V., 1992, Effective and effient global optimization for conceptual rainfall-runo models. Water Resource Research, 28, (4), 1015-1031.

Fonseca, C.M., Fleming, P.J., 1993, Genetic algorithms for multiobjective optimization: Formulation, discussion and generalization, Proc. $5^{\text {th }}$ Int. Conf. on Genetic Algorithms, vol. 93, San Mateo, 416-423.

Gajda-Zagórska, E., 2015, Adaptive population-based algorithms for solving single-and multiobjective inverse problems, PhD thesis, AGH University of Science and Technology, Kraków.

Gajda-Zagórska, E., Smołka, M., Schaefer, R., Pardo, D., Álvarez-Aramberri, J., 2015, Multi-objective hierarchic memetic solver for inverse parametric problems, Procedia Computer Science, 51, 974-983.

Gajda-Zagórska, E., Schaefer, R., Smołka, M., Pardo, D., Álvarez-Aramberri, J., 2017, Multi-objective memetic inverse solver reinforced by local optimization methods, Journal of Computational Science, 18, 85-94.

Gao, L., Calo, V.M., 2014, Fast isogeometric solvers for explicit dynamics, Computer Methods in Applied Mechanics and Engineering, 274, 19-41.

Gunawan, S., Azarm, S., 2005, Multi-objective robust optimization using a ensitivity region concept. Structural and Multidisciplinary Optimization, 29 (1), 50-60.

Isshiki, M., Sinclair, D., Kaneko, S., 2006, Lens design: Global optimization of both performance and tolerance sensitivity, International Optical Design, page TuA5, Optical Society of America, 2006. doi: 10.1364/IODC.2006.TuA5, URL http://www.osapublishing.org/abstract.cfm?URI=IODC2006-TuA5.

Jin, Y., 2005, A comprehensive survey of fitness approximation in evolutionary computation, Soft Computing, 9 (1), 53-59.

Jin, Y., Branke, J., 2005, Evolutionary optimization in uncertain environments - a survey, IEEE Transaction on Evolutionary Computation, 9, 303-317.

Łoś, M., Sawicki, J., Smołka, M., Schaefer, R., 2017, Memetic approach for irremediable ill-conditioned parametric inverse problems, Procedia Computer Science, 108C, 867876.

Miettinen, K., 1999, Nonlinear multiobjective optimization, Kluwer Academic Publishers, Boston.

Paruch, M., Majchrzak, E., 2007, Identification of tumor region parameters using evolutionary algorithm and multiple reciprocity boundary element method, Engineering Applications of Artificial Intelligence, 20 (5), 647-655. 
Petrone, G., Axerio-Cilies, J., Quagliarella, D.,Iaccarino, G., 2013, A probabilistic non-dominated sorting ga for optimization under uncertainty, Engineering Computations, 30, 1054-1085.

Sawicki, J., Łoś, M., Smołka, M., Schaefer, R., Julen ÁlvarezAramberri, J., 2018, Approximating landscape insensitivity regions in solving ill-conditioned inverse problems. Memetic Computing, doi: https://doi.org/10.1007/s12293-0180258-5.

Smołka, M., 2015, Memetic strategies and autonomous systems for solving inverse problems, Dissertations and Monographs, AGH University of Science and Technology Press, volume 311, Kraków.

Smołka, M., Gajda-Zagórska, E., Schaefer, R., Paszyński, M., Pardo, D., 2015a, A hybrid method for inversion of 3D AC logging measurements, Applied Soft Computing, 36, 422456.

Smołka, M., Schaefer, R., Paszyński, M., Pardo, D., ÁlvarezAramberri, J., 2015b, An agent-oriented hierarchic strategy for solving inverse problems. International Journal of Applied Mathematics and Computer Science, 25 (3), 483-498.

Tarantola, A., 2005, Inverse Problem Theory, Mathematics and its Applications, Society for Industrial and Applied Mathematics.

Tikhonov, A.N., Goncharsky, A.V., Stepanov, V.V., Yagola, A.G., 1995, Numerical methods for the solution of illposed problems, Springer-Verlag.

Ursem, R.K., 1999, Multinational evolutionary algorithms, Proc. Congress on Evolutionary Computation CEC'99, vol. 3, IEEE.

\section{ROZPOZNAWANIE NIEWRAŻLIWOŚCI \\ W WIELOKRYTERIALNYCH PROBLEMACH \\ ODWROTNYCH PRZY UŻYCIU ZLOŻONEJ STRATEGII EWOLUCYJNEJ}

\section{Streszczenie}

Artykuł prezentuje złożoną strategię rozwiązywania źle postawionych problemów odwrotnych sformułowanych jako wielokryterialne zadania optymalizacji globalnej. Opisana strategia umożliwia identyfikację obszarów niewrażliwości funkcji celu wokół spójnych składowych zbioru Pareto. Cel jest osiągany w dwu etapach. W pierwszym z nich — globalnym — składowe spójne zbioru Pareto są lokalizowane i separowane przy pomocy wielopopulacyjnej hierarchicznej strategii memetycznej HMS. W etapie drugim - lokalnym — przy użyciu specjalnie sprofilowanego procesu ewolucyjnego wykorzystującego operator selekcji wyborczej z wieloma zwycięzcami produkowana jest losowa próbka rozłożona jednostajnie na każdej składowej i jej bliskim otoczeniu. Finalnie każda lokalna próbka jest użyta jako baza

do zbudowania lokalnej aproksymacji funkcji dominacji. Zbiory poziomicowe tej aproksymacji dla odpowiednio niskich poziomów stanowią przybliżenie zbiorów niewrażliwości wokół składowych spójnych. Możliwości strategii zostały zweryfikowane przy użyciu specjalnie zaprojektowanych dwukryterialnych funkcji testowych. 